Canad. Math. Bull. Vol. 20 (1), 1977

\title{
AN ASYMPTOTIC ESTIMATE FOR THE BERNOULLI AND EULER NUMBERS
}

\author{
BY \\ DAVID J. LEEMING
}

1. Introduction. We derive here simple asymptotic estimates for both the Euler and Bernoulli numbers. The derivations follow easily from known results, but I am unable to find them elsewhere in the literature. C. Jordan [1, p. 245 and p. 303] gives some related inequalities. Other properties of these two classical sets of numbers may be found in [1], [3] and [4].

2. The Bernoulli case. It is well known (see e.g. [2]) that the Fourier series expansion of $B_{2 k}(x)$, the Bernoulli polynomial of degree $2 k$ is

$$
B_{2 k}(x)=\frac{(-1)^{k+1} 2(2 k) !}{(2 \pi)^{2 k}} \sum_{n=1}^{\infty} \frac{\cos 2 n \pi x}{n^{2 k}} \quad(k=1,2, \ldots)
$$

valid for $0 \leq x \leq 1$. Setting $x=\frac{1}{4}$ in (1) yields

Hence,

$$
B_{2 k}\left(\frac{1}{4}\right)=\frac{(-1)^{k+1} 2(2 k) !}{(4 \pi)^{2 k}} \sum_{n=1}^{\infty} \frac{(-1)^{n}}{n^{2 k}}
$$

$$
\left|B_{2 k}\left(\frac{1}{4}\right)\right| \sim \frac{2(2 k) !}{(4 \pi)^{2 k}} \quad(k \rightarrow \infty)
$$

Using Stirling's formula and the known result (see, e.g. [4], p. 22) $B_{2 k}\left(\frac{1}{4}\right)=$ $-2^{-2 k}\left(1-2^{1-2 k}\right) B_{2 k}$ we get

$$
\left|B_{2 k}\left(\frac{1}{4}\right)\right| \sim 4 \sqrt{ } \pi k\left(\frac{k}{2 \pi e}\right)^{2 k} \quad(k \rightarrow \infty)
$$

(Note that the coefficient $\sqrt{ } 8 \pi k$ appearing in [2], p. 537 is incorrect and should read $4 \sqrt{ } \pi k$ ).

Taking the $2 k$ th root in (3) yields

$$
\left|B_{2 k}\right|^{1 / 2 k} \sim \frac{k}{\pi e} \quad(k \rightarrow \infty) .
$$

A short table helps to illustrate the rate at which the two quantities are

Received by the editors May 24, 1976. 
approaching each other. Let $S_{k}=\left|B_{2 k}\right|^{1 / 2 k}$ and $T_{k}=k / \pi e$

\begin{tabular}{|r|c|c|c|}
\hline \multicolumn{1}{|c|}{$k$} & $S_{k}$ & $T_{k}$ & $S_{k} / T_{k}$ \\
\hline 5 & 0.77258 & 0.58550 & 1.31952 \\
10 & 1.36829 & 1.17100 & 1.16848 \\
15 & 1.96175 & 1.75649 & 1.11685 \\
20 & 2.55351 & 2.34199 & 1.09031 \\
25 & 3.14414 & 2.92749 & 1.07401 \\
30 & 3.73399 & 3.51299 & 1.06291 \\
\hline
\end{tabular}

3. The Euler case. The Fourier series expansion of $B_{2 k+1}(x)$, the Bernoulli polynomial of degree $2 k+1$ is

$$
B_{2 k+1}(x)=\frac{(-1)^{k+1} 2(2 k+1) !}{(2 \pi)^{2 k+1}} \sum_{n=1}^{\infty} \frac{\sin 2 n \pi x}{n^{2 k+1}} \quad(k=1,2, \ldots)
$$

valid for $0 \leq x \leq 1$. Setting $x=\frac{1}{4}$ in (5) yields

$$
B_{2 k+1}\left(\frac{1}{4}\right)=\frac{(-1)^{k} 2(2 k+1) !}{(2 \pi)^{2 k+1}} \sum_{n=1}^{\infty} \frac{(-1)^{n}}{(2 n-1)^{2 k+1}}
$$

Therefore,

$$
\left|B_{2 k+1}\left(\frac{1}{4}\right)\right| \sim \frac{2(2 k+1) !}{(2 \pi)^{2 k+1}} \quad(k \rightarrow \infty) .
$$

It is known (see e.g. [4], p. 29) that

$$
B_{2 k+1}\left(\frac{1}{4}\right)=\frac{-(2 k+1) E_{2 k}}{4^{2 k+1}} .
$$

Therefore, from (7) and (8) we get

$$
\left|E_{2 k}\right| \sim \frac{2^{2 k+2}(2 k) !}{\pi^{2 k+1}} \quad(k \rightarrow \infty)
$$

Using Stirling's formula in (9) we have

$$
\left|E_{2 k}\right| \sim \frac{8 \sqrt{ } k}{\sqrt{ } \pi}\left(\frac{4 k}{\pi e}\right)^{2 k} \quad(k \rightarrow \infty)
$$

Taking the $2 k$ th root in (10) yields

$$
\left|E_{2 k}\right|^{1 / 2 k} \sim \frac{4 k}{\pi e} \quad(k \rightarrow \infty)
$$

A second short table here illustrates the rate at which these two quantities are 
approaching each other. Let $w_{k}=\left|E_{2 k}\right|^{1 / 2 k}$ and $y_{k}=4 k / \pi e$.

\begin{tabular}{|r|c|c|c|}
\hline \multicolumn{1}{|c|}{$k$} & $w_{k}$ & $y_{k}$ & $w_{k} / y_{k}$ \\
\hline 5 & 2.95357 & 2.34199 & 1.26133 \\
10 & 5.35096 & 4.68399 & 1.14239 \\
15 & 7.73128 & 7.02598 & 1.10038 \\
20 & 10.0994 & 9.36797 & 1.07807 \\
25 & 12.4635 & 11.7100 & 1.06435 \\
30 & 14.8240 & 14.0520 & 1.05494 \\
\hline
\end{tabular}

\section{REFERENCES}

1. C. Jordan, Calculus of Finite Differences, Chelsea, NY, 1965.

2. D. H. Lehmer, On the maxima and minima of Bernoulli polynomials, American Math. Monthly, 47 (1940) 533-538.

3. L. M. Milne-Thomson, The Calculus of Finite Differences, Macmillan, London, 1951.

4. N. E. Nörlund, Vorlesungen Über Differenzenrechnung, Chelsea, NY, 1954.

DEPT OF MATH.

UNIVERSITY OF VICTORIA,

Victoria, B.C., CANADA 